\title{
DECIPHERING SINGULARITIES BY DISCRETE METHODS
}

\author{
YVES TOURIGNY AND MICHAEL GRINFELD
}

\begin{abstract}
We consider the problem of estimating numerically the parameters of singularities of solutions of differential equations. We propose a novel approach which is based on discretizing the governing equation and "timestepping" in the complex domain. Some applications to ordinary and partial differential equations are discussed.
\end{abstract}

\section{INTRODUCTION}

Many nonlinear differential problems feature local solutions which develop singularities. From a mathematical point of view, the question as to whether a singularity will occur is fundamental, since most global existence proofs are based upon extending the local solution. Further, the occurrence of singularities in mathematical models often has a physical interpretation (e.g., ignition in combustion, focusing in optics, cusps in free-surface flows, etc.), and it becomes important to determine the location and nature of the singularities, as well as their dependence on the data.

Numerical investigations of singularities can be useful in many contexts. They may provide a practical means of testing mathematical results or may serve as a heuristic tool in forming plausible conjectures. In addition, there are many examples in numerical analysis where the presence of singular points leads to a severe loss of computational efficiency. In such circumstances, much could be gained by making appropriate use of information on the troublesome singularities.

Following Sulem et al. [23], we may divide the methods which have been used for the detection and decipherment of singularities into two broad classes: those based upon expanding the solution in a Taylor series, using some known initial or boundary data, and those based upon discretizing the governing equation.

Methods based upon discretization offer great flexibility and are generally easy to implement. In the development of such methods, considerable thought must be given to the problem of extracting estimates for the singularity parameters from a sample of the values taken by the discrete solution. Sulem et al. [23] describe a technique which exploits the structure of the Fourier spectrum (in space) of the discrete solution. Tourigny and Sanz-Serna [24] obtain estimates via least squares fitting. In the latter case, the formulae for the singularity parameters are valid only asymptotically as the singularity is approached. In this

Received by the editor November 13, 1992.

1991 Mathematics Subject Classification. Primary 65L05, 65P05; Secondary 34A20. 
limit, it is necessary to adjust the mesh size so as to maintain the accuracy of the discretization. For this reason, the development of special adaptive techniques $[2,9]$ has received some attention.

By contrast, in techniques based on Taylor series, the accuracy of the estimates for the singularity parameters can, to some extent, be monitored "away" from the singularity by processing a sufficiently large number of Taylor coefficients. This approach has a sound mathematical basis [5, 6, 14]. However, its success depends upon the possibility of computing the coefficient sequence efficiently. Meiron et al. [19] give some indication of the computational effort required to generate Taylor coefficients in a nontrivial context.

Of course, in addition to the two classes described above, one may also envisage the development of "hybrid" methods. In [16], discretization formulae are proposed which incorporate the singularity parameters as additional unknowns. Estimates for the singularity parameters are then found by maximizing the order of consistency. As it turns out, the resulting estimates are identical to those obtained by Chang and Corliss [5] on the basis of Darboux's theorem. In order to avoid evaluating high-order derivatives, Shaw [22] proposed a modification of the basic method which requires divided differences instead.

In most applications, the independent variables have a physical meaning and are usually restricted to the real axis. However, for the purpose of investigating singularities, it is more fruitful, as anticipated by Corliss [6], to take a broader view and continue the solution along complex paths. In this work, we make systematic use of integration in the complex domain and show how this can assist in the numerical analysis of singularities. In our approach, the computational basis is provided by "classical" discretization schemes (notably those of the Runge-Kutta class). A link with methods of Taylor series type is provided by an algorithm due to Lyness [18] which, in essence, is a discrete version of Cauchy's integral formula for a circular contour. This enables the calculation of approximate Taylor coefficients via Fast Fourier Transform.

Those approximate Taylor coefficients may be used to locate the singular points and also to determine the order of poles [10]. In order to determine the nature of algebraic branch points, we propose a novel technique based on "numerical continuation". In short, this method yields estimates for the order by processing the variation in the discrete solution after it has been continued along a closed path surrounding the singularity.

The remainder of the paper is structured as follows: In $\S 2$, we briefly review Lyness's algorithm for the approximate calculation of Taylor coefficients. The application of this algorithm to the problem of locating singularities of solutions of differential equations is discussed in $\S 3$. In $\S 4$, we apply this technique to a semilinear parabolic equation and study the motion of complex singularities for a one-parameter family of initial data. In $\S 5$, we examine the problem of finding the order of branch points by numerical continuation. Finally, $\S 6$ is devoted to a summary of our conclusions.

\section{The CAlCUlation OF DisCRETE TAYLOR COEFFicientS}

In order to simplify the exposition, we shall, for the moment, restrict our attention to the scalar Cauchy problem

$$
\frac{d x}{d t}=f(x, t), \quad x(0)=x_{0},
$$


where $f$ is analytic at $\left(x_{0}, 0\right)$. The solution $x$ may be represented by a Taylor series

$$
x(t)=\sum_{n=0}^{\infty} a_{n} t^{n}, \quad|t|<R,
$$

where $R$ is the radius of convergence. The nearest singularities of $x$ lie on the circle of convergence. If, in addition, we assume that $x_{0}$ is real and that $f$ is real for real values of $x$ and $t$, then the singularities are either on the real axis, or else occur as complex conjugate pairs. In either case, the location of the singularities may be estimated in terms of the sequence $\left\{a_{n}\right\}$ (cf. $\S 3$ ).

Brent and Kung [3] describe an algorithm applicable to a large class of ordinary differential equations which can, in principle, generate the first $n$ Taylor coefficients in $O(n \log n)$ operations. This algorithm is based on linearizing the differential equation via Newton's method. It uses the Fast Fourier Transform in order to compute products of polynomials. However, the practical use of this algorithm is limited by the fact that a sufficient (a priori unknown) number of the first coefficients must be available initially in order to guarantee the convergence of Newton's iteration. The algorithm implemented by Corliss and Chang [8] is based on Leibniz's rule and computes the first $n$ coefficients in $O\left(n^{2}\right)$ operations. The algorithms used in symbolic packages such as MAPLE, REDUCE, or MACSYMA are generally impractical unless the function $f$ in (2.1) is particularly simple.

In terms of flexibility and ease of implementation, it may be more profitable to calculate approximate rather than exact coefficients. In the remainder of this section, we review an algorithm due to Lyness [18].

Cauchy's integral formula applied to a circular contour of radius $r<R$ centered at the origin yields

$$
a_{n}=\frac{1}{r^{n}}\left[\frac{1}{2 \pi} \int_{0}^{2 \pi} x\left(r e^{i \theta}\right) e^{-i n \theta} d \theta\right] .
$$

The expression within brackets is the $n$th Fourier coefficient of the periodic function $x\left(r e^{i \theta}\right)$. Lyness [18] suggests the approximation

$$
a_{n}^{(N)}=\frac{1}{r^{n}}\left[\frac{1}{N} \sum_{j=0}^{N-1} x\left(r e^{i \theta_{j}}\right) e^{-i n \theta_{j}}\right]
$$

for $a_{n}$, where $\theta_{j}=2 \pi j / N$. The expression within brackets in (2.3) is a discrete Fourier coefficient of $x\left(r e^{i \theta}\right)$. Thus, the $N$ approximate Taylor coefficients $a_{n}^{(N)}, n=0,1, \ldots, N-1$, may be computed in $O(N \log N)$ operations via Fast Fourier Transform.

The formula (2.3) requires the evaluation of the solution $x$ at equally spaced points along the circle of radius $r$ centered at the origin. The algorithm can therefore be used in conjunction with time-stepping procedures. Assuming that a method has been selected for the discretization of (2.1), we define the discrete Taylor coefficient $a_{n}^{h}$ via

$$
r^{n} a_{n}^{h}=\frac{1}{N} \sum_{j=0}^{N-1} x_{j}^{h} e^{-i n \theta_{j}}
$$


where $x_{j}^{h}$ is the discrete approximation to $x\left(r e^{i \theta_{j}}\right)$.

We now discuss the accuracy of this procedure. We have

$$
r^{n} a_{n}-r^{n} a_{n}^{h}=\left(r^{n} a_{n}-r^{n} a_{n}^{(N)}\right)+\left(r^{n} a_{n}^{(N)}-r^{n} a_{n}^{h}\right) .
$$

By standard approximation theory,

$$
r^{n} a_{n}^{(N)}-r^{n} a_{n}=\sum_{k=1}^{\infty} r^{n+k N} a_{n+k N}, \quad 0 \leq n \leq N-1 .
$$

Thus, the difference between $a_{n}^{(N)}$ and $a_{n}$ depends on the decay rate of the Taylor coefficients of $x$. Cauchy's estimate yields

$$
\left|r^{n} a_{n}^{(N)}-r^{n} a_{n}\right| \leq \frac{\lambda^{n+N}}{1-\lambda^{N}} \max _{0 \leq \theta \leq 2 \pi}\left|x\left(\rho e^{i \theta}\right)\right|, \quad 0 \leq n \leq N-1,
$$

where $\lambda=r / \rho$ and $\rho$ is any positive number in $(r, R)$. This shows that the error in approximating $a_{n}$ by $a_{n}^{(N)}$ ( $n$ and $r$ fixed) decays exponentially as $N$ increases.

It is also easily seen that, if $p$ is the order of consistency of the discretization employed, then

$$
\left|r^{n} a_{n}^{(N)}-r^{n} a_{n}^{h}\right| \leq C_{r, p} h^{p},
$$

where $C_{r, p}$ is a constant independent of $n$ and $h$ which, roughly speaking, is the size of $x^{(p+1)}$ along the circle parametrized by $r e^{i \theta}$. In practice, the error due to the discretization clearly dominates in (2.5).

\section{LOCATING SINGULARITIES: FIRST EXAMPLE}

The discrete Taylor coefficients may be used to locate singularities. For the purpose of illustration, consider the model problem

$$
\frac{d x}{d t}=x^{3}(1-t), \quad x(0)=x_{0},
$$

where $x_{0}$ is real. The solution

$$
x(t)=\left(1 / x_{0}^{2}-2 t+t^{2}\right)^{-1 / 2}
$$

has algebraic branch points at

$$
t_{*}=1 \pm\left(1-1 / x_{0}^{2}\right)^{1 / 2} .
$$

(For $x_{0}=1$, the two branch points merge into a pole.) Thus, $x$ has no real singularities if $\left|x_{0}\right|<1$. This situation is typical of many differential problems for which global existence for $t>0$ is only guaranteed if the size of the initial datum is below a certain threshold [17]. We use the classical RungeKutta method [4] for the discretization of (3.1). This is a fourth-order explicit scheme.

We begin with the case $x_{0}>1$. This gives rise to a real singularity located at

$$
t_{*}=R=1-\left(1-1 / x_{0}^{2}\right)^{1 / 2} .
$$

The parameter $R$ may be estimated from the following formulae [5, 14]:

$$
\begin{gathered}
\frac{1}{R}=\frac{a_{n}}{a_{n-1}}+O\left(n^{-1}\right) \quad(\text { ratio test }), \\
\frac{1}{R}=n \frac{a_{n}}{a_{n-1}}-(n-1) \frac{a_{n-1}}{a_{n-2}}+O\left(n^{-2}\right) \quad(\text { three-term test }) .
\end{gathered}
$$


Replacing $a_{n}$ by $a_{n}^{h}$ in (3.2) and (3.3), we obtain discrete estimates of $R$. In Table 1 , the results obtained by using discrete and exact Taylor coefficients in each test is summarized. These calculations, as all those reported in this paper, were carried out on a network of Sun computers using double-precision complex arithmetic. In the present case, the value $r=.2$ was used. The quantity $h=1 / N$ denotes the reciprocal of the number of steps used to march along the circle.

TABLE $1 . x_{0}=\sqrt{2}, R=.29289322$

\begin{tabular}{c|ccc|ccc}
\hline$n$ & \multicolumn{3}{|c|}{ Ratio test } & \multicolumn{3}{c}{ Three-term test } \\
& Exact & $h=2^{-8}$ & $h=2^{-9}$ & Exact & $h=2^{-8}$ & $h=2^{-9}$ \\
\hline 10 & .30851892 & .30851895 & .30851892 & .29261669 & .29261681 & .29261670 \\
20 & .30044749 & .30044784 & .30044751 & .29284385 & .29284516 & .29284393 \\
40 & .29661094 & .29661430 & .29661113 & .29288247 & .29289637 & .29288318 \\
80 & .29473777 & .29452297 & .29522056 & .29289070 & .33463291 & .40358805 \\
\hline
\end{tabular}

These results, and more particularly those corresponding to $n=80$, are best explained in terms of the relative error

$$
e_{n}^{h}=\frac{r^{n} a_{n}^{h}-r^{n} a_{n}}{r^{n} a_{n}}
$$

incurred in approximating $r^{n} a_{n}$. We have

$$
\frac{a_{n}^{h}}{a_{n-1}^{h}}=\frac{a_{n}}{a_{n-1}} \frac{1+e_{n}^{h}}{1+e_{n-1}^{h}} .
$$

Thus, we see that in order to approximate the ratio $a_{n} / a_{n-1}$ to a good accuracy, we need to select $h$ and $r$ in such a way that

$$
\left|e_{n-1}^{h}\right|,\left|e_{n}^{h}\right| \leq \varepsilon,
$$

where $\varepsilon$ is a given tolerance. Assuming that the error due to the discretization dominates in (2.5), we are led to the requirement

$$
C_{r, p} h^{p} \leq \varepsilon r^{n}\left|a_{n}\right| \leq \varepsilon(r / R)^{n},
$$

approximately for large $n$, using Hadamard's formula. This shows that, for a fixed value of $r<R$, only $O\left(p \log \frac{1}{h}\right)$ of the ratios may be approximated to a good accuracy as $h \rightarrow 0$. As a consequence, if the formulae (3.2) and (3.3) are used for the approximation of $R$, then the procedure described above converges extremely slowly as the stepsize is reduced.

In some instances, however, it is possible to obtain estimates for $R$ which converge at the rate $O\left(h^{p}\right)$. For the particular problem (3.1), this can be achieved by applying the ratio test to the logarithmic derivative of $x$ rather than to $x$ itself [1]. Indeed, note that the derivative of $\log x(t)$ has a pole at $t=R$, and thus

$$
\frac{1}{R}=\frac{b_{n}}{b_{n-1}}+O\left(\mu^{n}\right) \quad(\mathrm{d}-\log \text { ratio test })
$$

where $b_{n}$ denotes the $n$th Taylor coefficient of $(d x / d t) / x$, and $0<\mu<1$ is the ratio of the distances to the two branch points. In the context of Lyness's 
algorithm, this idea is easily implemented. We simply set

$$
r^{n} b_{n}^{h}=\frac{1}{N} \sum_{j=0}^{N-1} \frac{f\left(x_{j}^{h}, r e^{i \theta_{j}}\right)}{x_{j}^{h}} e^{-i n \theta_{j}}
$$

where $\theta_{j}$ and $x_{j}^{h}$ are just as in (2.4), and where $f(x, t)$ is the right-hand side of the first equality in (3.1). The results of numerical computations with $r=.2$ are displayed in Table 2.

TABLE 2. D-log ratio test. $x_{0}=\sqrt{2}, R=.29289322$

\begin{tabular}{r|ccccc}
\hline$n$ & Exact & $h=2^{-6}$ & $h=2^{-7}$ & $h=2^{-8}$ & $h=2^{-9}$ \\
\hline 5 & .29292929 & .29292901 & .29292928 & .29292929 & .29292929 \\
10 & .29289322 & .29289773 & .29289350 & .29289324 & .29289323 \\
15 & .29289322 & .29291568 & .29289447 & .29289329 & .29289322 \\
20 & .29289322 & .29296250 & .29289658 & .29289341 & .29289323 \\
\hline
\end{tabular}

It should be pointed out that the result of applying the ratio test to the logarithmic derivative of $x$ is not always as spectacular as in the present case. The estimate (3.5) is valid for solutions of the form

$$
x(t)=\left(1-t / t_{*}\right)^{-\nu} y(t),
$$

where $y$ is analytic in a disk of radius greater than $\left|t_{*}\right|$. However, the solutions of even relatively simple ordinary differential equations often have a more complicated structure (cf. $\S 5$ ). Nevertheless, the d-log ratio test often improves upon the accuracy of the ratio test by a power of $n^{-1}$.

We now turn to the case $\left|x_{0}\right|<1$. This gives rise to complex conjugate singularities and, in this case, the previous formulae will lead to oscillating divergent sequences. For singularities located at $t_{*}=R e^{ \pm i \theta}$, the formulae (four-term test)

$$
R^{2} \approx \frac{a_{n-2}^{2}-a_{n-3} a_{n-1}}{a_{n-1}^{2}-a_{n} a_{n-2}}, \quad 2 R \cos \theta \approx \frac{a_{n-2} a_{n-1}-a_{n-3} a_{n}}{a_{n-1}^{2}-a_{n-2} a_{n}}
$$

may be used. Note that these formulae are exact when applied to the logarithmic derivative of $x$. The results of sample computations with $x_{0}=\sqrt{10 / 11}$ and $r=.6$ are summarized in Table 3 , where estimates for the imaginary part of $t_{*}$ are shown.

$$
\text { TABLE 3. } x_{0}=\sqrt{\frac{10}{11}}, R \sin \theta=.31622777
$$

\begin{tabular}{rccc|rccc}
\hline & \multicolumn{3}{c|}{ Four-term test } & \multicolumn{3}{c}{ D-log four-term test } \\
$n$ & Exact & $h=2^{-9}$ & $h=2^{-10}$ & $n$ & Exact & $k=2^{-9}$ & $k=2^{-10}$ \\
\hline 5 & .25148727 & .25148726 & .25148727 & 5 & .31622777 & .31622776 & .31622777 \\
10 & .31260602 & .31260601 & .31260602 & 10 & .31622777 & .31622775 & .31622777 \\
20 & .31980348 & .31980362 & .31980349 & 20 & .31622777 & .31622780 & .31622777 \\
40 & .31904765 & .31904920 & .31904887 & 40 & .31622777 & .31622780 & .31622777 \\
\hline
\end{tabular}




\section{Application to a SEMilinear Parabolic PROBlem}

It is natural to ask whether the approach described in the previous section may also be used in the context of partial differential equations. This is indeed the case provided that the solution enjoys some analyticity properties in a suitable region of the complex domain. One possible line of development is to complexify each independent variable and deal with multiple power series. In this respect, note that a generalization of Cauchy's integral formula is available in several complex variables. Thus, in principle, multiple Fast Fourier Transforms enable the calculation of approximate Taylor coefficients as before. However, before this program can be carried out, one requires a means of "numerically continuing" the solution in the complex hyperplane. Further, in several variables, some theoretical work is needed in order to determine the relationship between the Taylor coefficients and the singularity parameters.

For evolution problems, a simpler expedient is to reduce the problem to a system of ordinary differential equations via spatial discretization. In this section, we apply this procedure to the model problem

$$
\begin{gathered}
\frac{\partial u}{\partial t}=\frac{\partial^{2} u}{\partial x^{2}}+u^{2}, \quad(x, t) \in(0,1) \times \mathbf{C}_{+}, \\
u(0, t)=u(1, t)=0, \quad t \in \mathbf{C}_{+}, \\
u(x, 0)=\alpha \sin \pi x, \quad x \in[0,1],
\end{gathered}
$$

where $\mathbf{C}_{+}$is the set of complex numbers with positive real part and $\alpha$ is a real positive parameter. This problem and its generalizations have received considerable attention in the literature $[17,21]$. In this section, we propose to trace numerically the motion of the singularities in the complex plane as the parameter $\alpha$ varies. This type of question is important in many contexts, but is still beyond the scope of rigorous analytical methods [15] . Nakagawa [20] has studied this problem by a finite difference method with a variable time-step and has proved the convergence of an estimate for the location of real positive singularities.

For illustrative purposes, we have chosen the spatial discretization

$$
\frac{d u_{j}}{d t}=\frac{1}{\Delta x^{2}}\left(u_{j-1}-2 u_{j}+u_{j+1}\right)+u_{j}^{2}, \quad j=1, \ldots, J
$$

where $\Delta x=1 /(J+1)$ and $u_{j}(t)$ denotes an approximation to $u(j \Delta x, t)$. In the above expression, $u_{0}(t)=u_{J+1}(t) \equiv 0$. The time-stepping algorithm used is a fourth-order implicit Runge-Kutta method characterized by the table [4]

\begin{tabular}{ccc|c}
$\gamma$ & 0 & 0 & $\gamma$ \\
$1 / 2-\gamma$ & $\gamma$ & 0 & $1 / 2$ \\
$2 \gamma$ & $1-4 \gamma$ & $\gamma$ & $1-\gamma$ \\
\hline$\beta$ & $1-2 \beta$ & $\beta$ &
\end{tabular}

where $\gamma=1 / 2+1 / \sqrt{3} \cos (\pi / 18)$ and $\beta=\left[6(1-2 \gamma)^{2}\right]^{-1}$. As in the previous section, $h$ denotes the reciprocal of the number of steps.

In order to locate the singularities, we have chosen to expand $u_{j}(t)$ with $j=(J+1) / 2$. Alternatively, one could expand a suitable functional such as, for instance, the discrete counterpart of $\int_{0}^{1} u^{2}(x, t) d x$. This would present no 
difficulty, since all that is required is the evaluation of the given quantity on a circle in the complex domain. In choosing the center and radius of the circle, one should however be careful to avoid the imaginary axis which, for the partial differential problem, constitutes the left-hand boundary of the region where $u$ is expected to be analytic. Thus, we are interested only in the nearest singular points with strictly positive real part.

For a fixed value of the meshsize $\Delta x$, we may locate the singularities by choosing a suitable point of expansion, and adjusting $r$ and $h$ appropriately. In our computations, we have observed that it is necessary to expand very near the singular point in order to ensure that the ratios $a_{n} / a_{n-1}$ are accurately computed. This requirement becomes more severe as $\Delta x$ is reduced. The implementation only catered for points of expansion situated on the real axis. This made it difficult to accurately locate complex conjugate pairs of singularities since, in this case, only the first few Taylor coefficients could be well approximated. For instance, in the case $\Delta x=1 / 20, \alpha=11.48$, and $r=10^{-3}$, only the first six coefficients could be relied upon, as indicated in Table 4.

TABLE 4. Four-term test for the imaginary part of the singular point nearest to $t=.7$

\begin{tabular}{c|cccccc}
\hline$n$ & $h=2^{-6}$ & $h=2^{-7}$ & $h=2^{-8}$ & $h=2^{-9}$ & $h=2^{-10}$ & $h=2^{-11}$ \\
\hline \hline 3 & .35615107 & .35652587 & .35657155 & .35657663 & .35657715 & .35657717 \\
4 & .34899790 & .34858438 & .34850191 & .34848921 & .34848772 & .34848793 \\
5 & .36825079 & .32551869 & .31960534 & .31883033 & .31869438 & .31879080 \\
6 & - & .30296600 & .31784852 & .32192211 & .32011844 & .31668660 \\
\hline
\end{tabular}

However, bearing in mind that the singularities for (4.4) should resemble poles, the number of coefficients obtained was still sufficient to guarantee two correct digits after the decimal point. Our findings are summarized qualitatively in Figure 1 and quantitatively in Table 5.

TABLE 5. Estimated location of the singularities

\begin{tabular}{c|ccccc}
\hline$\alpha$ & 11.40 & 11.45 & 11.46 & 11.47 & 11.48 \\
$t_{*}$ & $.48 \pm .32 i$ & $.57 \pm .32 i$ & $.58 \pm .32 i$ & $.64 \pm .32 i$ & $.72 \pm .32 i$ \\
\hline$\alpha$ & 11.488842 & 11.488843 & 11.488844 & 11.488845 & 11.488846 \\
$t_{*}$ & $1.53 \pm 32 i$ & $1.57 \pm .32 i$ & $1.66 \pm .32 i$ & 1.76 & 1.60 \\
\hline$\alpha$ & 11.4889 & 11.49 & 11.495 & 11.5 & 11.6 \\
$t_{*}$ & 1.36 & .92 & .75 & .69 & .46 \\
\hline
\end{tabular}

The results suggest the existence of a critical value $\alpha_{\text {crit }}$ such that, for $\alpha<\alpha_{\text {crit }}$, the singularities move along an imaginary line. For $\alpha>\alpha_{\text {crit }}$, the nearest singularity moves towards the origin along the real axis. Interestingly, the trajectory of the complex singularities does not join smoothly with the real axis as $\alpha \rightarrow \alpha_{\text {crit }}$

Naturally, those findings apply only to the semidiscretization (4.4) with the particular choice $\Delta x=1 / 20$. Indeed, for a fixed value of $\alpha$, the position of the nearest singularity will alter with the meshsize. As Table 6 indicates, however, a limiting value is achieved as $\Delta x \rightarrow 0$. 
TABLE 6. $t_{*}$ as a function of the meshsize $\Delta x(\alpha=11.6)$

\begin{tabular}{c|cccccc}
\hline$\Delta x$ & $10^{-1}$ & $20^{-1}$ & $30^{-1}$ & $40^{-1}$ & $50^{-1}$ & $60^{-1}$ \\
$t_{*}$ & .4111 & .4624 & .4759 & .4810 & .4836 & .4849 \\
\hline
\end{tabular}

Computations performed with the meshsize $\Delta x=1 / 30$ have in fact confirmed the qualitative picture presented in Figure 1.

Finally, let us mention that a spatial discretization based on a spectral Galerkin method with sines as basis functions would very probably have enabled a much more accurate resolution. Indeed, Professor C. Hunter [13] pointed out that the single-mode approximation

$$
u(x, t) \approx U(t) \sin \pi x
$$

leads to the ordinary differential equation

$$
\frac{d U}{d t}=-\pi^{2} U+\frac{8}{3 \pi} U^{2}, \quad U(0)=\alpha .
$$

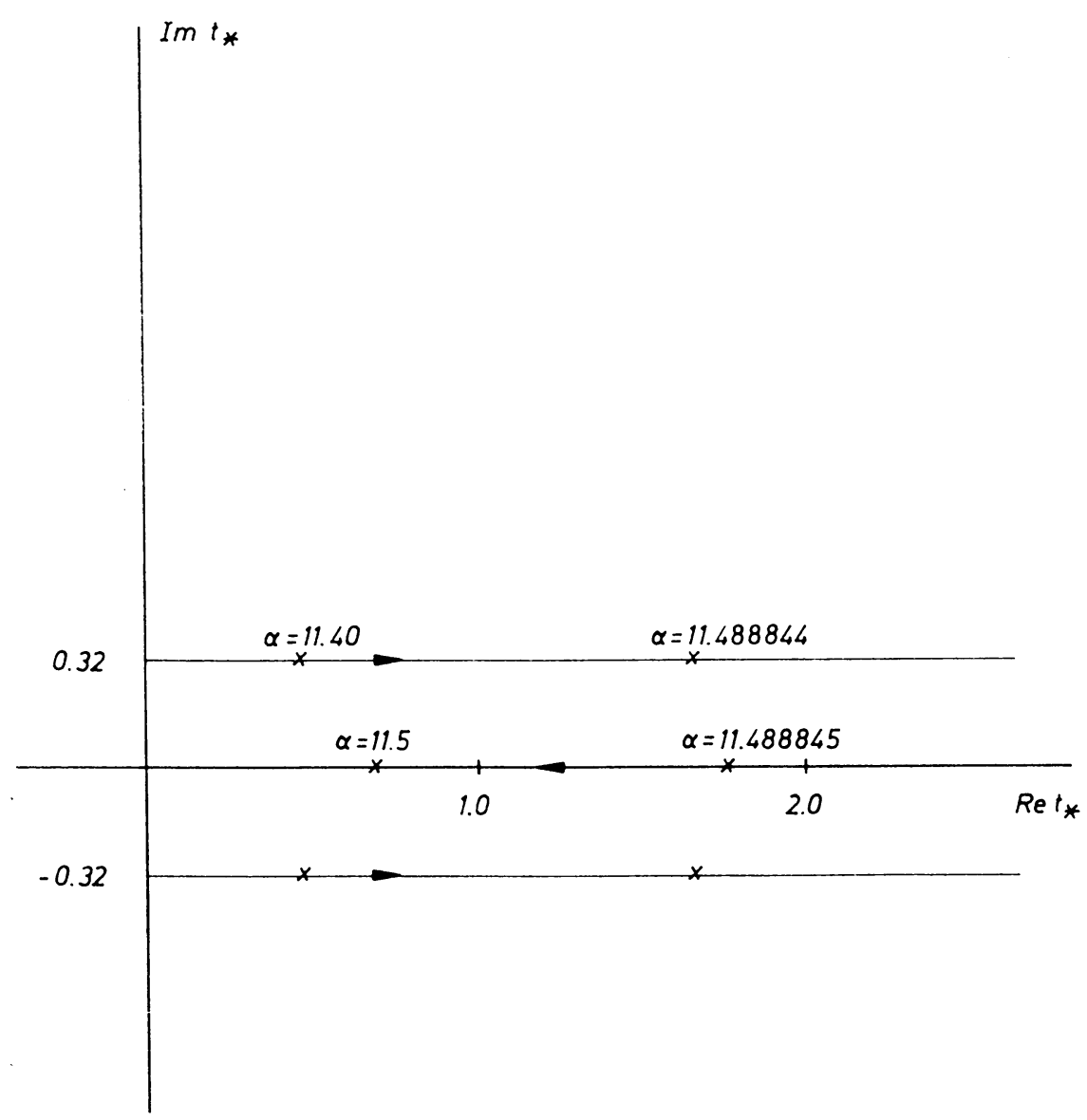

Figure 1 
After integration, it is readily seen that $U$ has a pole at

$$
t_{*}=-\frac{1}{\pi^{2}} \log \left(1-\frac{3 \pi^{3}}{8 \alpha}\right) \text {. }
$$

This yields $\alpha_{\text {crit }} \approx 3 \pi^{3} / 8 \approx 11.63$. Further, for $\alpha<3 \pi^{3} / 8$, the nearest singular points are given by the complex conjugate pair

$$
-\frac{1}{\pi^{2}} \log \left(\frac{3 \pi^{3}}{8 \alpha}-1\right) \pm \frac{1}{\pi} i
$$

Since $1 / \pi \approx 0.318$, this is in remarkable agreement with our finite-difference calculation using 19 grid-points. Clearly, the transition from complex to real singularities in this problem deserves further study.

\section{THE ORDER OF BRANCH POINTS}

We now examine the problem of determining the nature of the singularities. The special case of polar singularities is a classical topic discussed by Hadamard at the turn of the century. We refer to Henrici [10] for a detailed exposition. In this paper, we shall concentrate on algebraic branch points. The basic principle can also be applied to logarithmic branch points.

In principle, the order of branch points may be extracted from the sequence of Taylor coefficients. The formulae derived by Chang and Corliss [5] and Hunter and Guerrieri [14] are such that the location and order parameters are coupled. As a rule of thumb, the error in estimating the order is usually larger by a factor $O(n)$ than the error in estimating the location. Such formulae for the order often involve powers of $n$ as factors, and this tends to amplify any error incurred in the approximation of the Taylor coefficients.

In this section, we describe an alternative approach based on "numerical continuation". In order to motivate this approach, consider the simple case in which the solution $x$ takes the form

$$
x(t)=\left(t_{*}-t\right)^{-\gamma}
$$

where $\gamma$ is not an integer. Pose $x\left(t_{0}\right)=x_{0}$. If $x$ is continued along a simple closed curve surrounding the singular point, then its value after completing one circuit is

$$
x_{1}=e^{-i 2 \pi \gamma} x_{0}
$$

and thus

$$
\gamma=\frac{1}{2 \pi i} \log \left(x_{0} / x_{1}\right) \text {. }
$$

This suggests that we can obtain estimates for the order of an algebraic branch point by numerically integrating in the complex domain along closed curves. There are only two restrictions to observe: first, the branch point should be the only singularity enclosed by the curve; second, the discretization scheme should be consistent with the concept of Weierstrassian continuation. In particular, for evolution problems, the effect of taking one "time-step" should be equivalent to evaluating the solution from a truncated power series. Runge-Kutta methods obviously satisfy this requirement. One of the advantages of this technique is that the estimate for $\gamma$ is, to some extent, unaffected by small inaccuracies in locating the singularity. 
If the singularities arising from differential equations were of the simple form (5.1), then the estimate (5.2) would be exact to within the discretization error. Save in exceptional cases, however, $(5.1)$ is only valid asymptotically as $t \rightarrow t_{*}$. The structure of the solution in the neighborhood of the branch point may be much more complicated. To give but a simple example, consider the case where the right-hand side $f$ in the first equality in (2.1) is a rational function of $x$ with coefficients algebraic in $t$. Assume that the numerator and denominator are of degree $l$ and $m$, respectively, with $l-m>2$. Following Hille [12], we may show that branch points exist for suitable values of $x_{0}$, and that

$$
x(t)=\sum_{j=-1}^{\infty} c_{j}\left(t_{*}-t\right)^{j \gamma}
$$

for $\left|t-t_{*}\right|<\rho$, where $\rho$ is some positive constant and $\gamma=1 /(l-m-1)$. With this representation, we see that (5.2) should be replaced by

$$
\gamma=\frac{1}{2 \pi i} \log \left(x_{0} / x_{1}\right)+O\left(R^{\gamma}\right)
$$

where $R=t_{*}-t_{0}$. Thus, we can estimate $\gamma$ accurately only as the singularity is approached. Further, for strong nonlinearities, i.e., for $\gamma$ small, the convergence of this estimate is very slow as $R \rightarrow 0$.

It turns out that the fractional power series development (5.3) is typical of functions with an algebraic branch point. Without knowledge of $\gamma$, it is possible to use this information to retrieve $\gamma$ with increasing accuracy by winding around the branch point a sufficient number of times.

Let $x_{n}$ be the value at $t_{0}$ obtained after continuing $x$ by winding around the singular point $n$ times. Pose

$$
\omega=e^{i 2 \pi \gamma}, \quad a_{j}=c_{j} R^{j \gamma}\left(\omega^{j}-1\right), \quad \text { and } \quad \Delta_{n}=x_{n+1}-x_{n} .
$$

We have

$$
\Delta_{n}=a_{-1} \omega^{-n}+a_{1} \omega^{n}+a_{2} \omega^{2 n}+\cdots, \text { for } n=0,1, \ldots
$$

We may also write

$$
\begin{aligned}
\Delta_{0} & =a_{-1}+a_{1}+\cdots+a_{N-1}+O\left(R^{N \gamma}\right), \\
\Delta_{1} & =a_{-1} \omega^{-1}+a_{1} \omega+\cdots+a_{N-1} \omega^{N-1}+O\left(R^{N \gamma}\right), \\
& \vdots \\
\Delta_{N} & =a_{-1} \omega^{-N}+a_{1} \omega^{N}+\cdots+a_{N-1} \omega^{N(N-1)}+O\left(R^{N \gamma}\right) .
\end{aligned}
$$

For given $\Delta_{0}, \ldots, \Delta_{N}$, we may form a closed system of equations for the unknowns $a_{-1}, a_{1}, \ldots, a_{N-1}$, and $\omega$ by ignoring the terms of order $O\left(R^{N \gamma}\right)$ in (5.6). In order to estimate $\omega$, we may use Prony's method [11]. Let

$$
p_{N}(x)=z^{N}-\alpha_{N-1} z^{N-1}-\cdots-\alpha_{0}
$$

be the polynomial with roots $\omega^{-1}, \omega, \ldots, \omega^{N-1}$. We can obtain estimates for the coefficients $\alpha_{0}, \ldots, \alpha_{N-1}$ as follows: multiply the first equality in (5.6) by $-\alpha_{0}$, the second by $-\alpha_{1}$, etc. up to the penultimate equality and add to the last equality to obtain

$$
\Delta_{N}=\alpha_{0} \Delta_{0}+\alpha_{1} \Delta_{1}+\cdots+\alpha_{N-1} \Delta_{N-1}+O\left(R^{N \gamma}\right) .
$$


A similar equation is obtained if we consider $\Delta_{1}, \ldots, \Delta_{N-1}$ instead. By continuing this process, we arrive at

$$
\Delta_{N}=\alpha_{0} \Delta_{0}+\alpha_{1} \Delta_{1}+\cdots+\alpha_{N-1} \Delta_{N-1}+O\left(R^{N \gamma}\right),
$$

$$
\Delta_{2 N-1}=\alpha_{0} \Delta_{N-1}+\alpha_{1} \Delta_{N}+\cdots+\alpha_{N-1} \Delta_{2 N-1}+O\left(R^{N \gamma}\right) .
$$

With the terms of order $O\left(R^{N \gamma}\right)$ ignored, (5.8) reduces to a linear system which, if nonsingular, yields approximations $\tilde{\alpha}_{j}$ of the coefficients $\alpha_{j}$ of $p_{N}$.

For example, in the case $N=1,(5.8)$ reduces to

$$
\Delta_{1}=\alpha_{0} \Delta_{0}+O\left(R^{\gamma}\right) \text {. }
$$

The root of $z-\tilde{\alpha}_{0}$ provides an estimate for $\omega^{-1}$, and we are led to the formula

$$
\omega^{-1}=\frac{x_{2}-x_{1}}{x_{1}-x_{0}}+O\left(R^{2 \gamma}\right) \text {. }
$$

This formula is exact if $\gamma=1 / 2$. In the case $N=2,(5.8)$ becomes

$$
\begin{aligned}
& \Delta_{2}=\alpha_{0} \Delta_{0}+\alpha_{1} \Delta_{1}+O\left(R^{2 \gamma}\right), \\
& \Delta_{3}=\alpha_{0} \Delta_{1}+\alpha_{1} \Delta_{2}+O\left(R^{2 \gamma}\right) .
\end{aligned}
$$

The resulting system is nonsingular unless $\gamma$ is a multiple of $1 / 2$. The roots of $z^{2}-\tilde{\alpha}_{1} z-\tilde{\alpha}_{0}$ provide estimates for $\omega^{-1}$ and $\omega$, and we obtain a formula in terms of $x_{0}, \ldots, x_{4}$ which is exact for $\gamma=1 / 3$. More generally, it is straightforward to show that this procedure is exact for $\gamma=1 /(N+1)$. In this respect, the main observation is that Eq. (5.5) then involves no coefficients which have multiples of $N+1$ as subscripts.

Example 5.1. We consider the problem

$$
\frac{d x}{d t}=(x+t) x^{2}, \quad x(0)=.5 .
$$

This leads to a real singularity at $t_{*}=1.2105092$. In order to estimate $\gamma$, we integrate numerically along a circle of radius $R$ centered at $t_{*}$. We emphasize that this particular choice of contour is by no means the only possible one. It is only used to demonstrate the convergence properties of the procedure. The stepsize is such that the discretization error may be disregarded. The results obtained are shown in Table 7. The case $N=0$ corresponds to the estimate (5.2). Note that, as expected, the error for the case $N=0$ decays like $O(\sqrt{R})$.

TABLE 7. Estimates for the order of the singularity $(\gamma=1 / 2)$

\begin{tabular}{c|cc|cc}
\hline & \multicolumn{2}{|c|}{$N=0$} & \multicolumn{2}{c}{$N=1$} \\
$R$ & $\operatorname{Re} \gamma$ & $\operatorname{Im} \gamma$ & $\operatorname{Re} \gamma$ & $\operatorname{Im} \gamma$ \\
\hline 1 & $.50000000 e+0$ & $.89638197 e-1$ & $.50000000 e+0$ & $-.21203698 e-15$ \\
$2^{-1}$ & $.50000000 e+0$ & $.95781585 e-1$ & $.50000000 e+0$ & $-.53009245 e-15$ \\
$2^{-2}$ & $.50000000 e+0$ & $.79329707 e-1$ & $.50000000 e+0$ & $-.27564807 e-14$ \\
$2^{-3}$ & $.50000000 e+0$ & $.60190474 e-1$ & $.50000000 e+0$ & $-.40993816 e-14$ \\
$2^{-4}$ & $.50000000 e+0$ & $.44011445 e-1$ & $.50000000 e+0$ & $-.59016959 e-14$ \\
$2^{-5}$ & $.50000000 e+0$ & $.31646490 e-1$ & $.50000000 e+0$ & $-.92589481 e-14$ \\
\hline
\end{tabular}


Example 5.2. Consider the problem

$$
\frac{d x}{d t}=(x+t) x^{5}, \quad x(0)=.5 .
$$

A real singularity is located at $t_{*}=2.2864$. The error incurred for the cases $N=0,1,2$, and 3 are displayed in Table 8 , together with the observed local rate of convergence as $R \rightarrow 0$. This is calculated by taking the logarithm of the ratio of successive errors and dividing by $\log 2$.

TABLE 8. Decay of the error $\left(\gamma=\frac{1}{5}\right)$

\begin{tabular}{c|cc|cc|cc|cc}
\hline & \multicolumn{2}{|c|}{$N=0$} & \multicolumn{2}{|c|}{$N=1$} & \multicolumn{2}{c}{$N=2$} & \multicolumn{2}{c}{$N=3$} \\
$R$ & Error & Rate & Error & Rate & Error & Rate & Error & Rate \\
\hline 1 & $.35856 e-1$ & - & $.514855 e-1$ & - & $.571157 e-1$ & - & $.483825 e-1$ & - \\
$2^{-1}$ & $.356372 e-1$ & .01 & $.499021 e-1$ & .04 & $.566304 e-1$ & .01 & $.473218 e-1$ & .03 \\
$2^{-2}$ & $.345054 e-1$ & .05 & $.479263 e-1$ & .06 & $.564901 e-1$ & .00 & $.453104 e-1$ & .06 \\
$2^{-3}$ & $.329129 e-1$ & .07 & $.443135 e-1$ & .11 & $.560674 e-1$ & .01 & $.426686 e-1$ & .09 \\
$2^{-4}$ & $.310526 e-1$ & .08 & $.386535 e-1$ & .20 & $.269176 e-1$ & 1.06 & $.710014 e-2$ & 2.59 \\
$2^{-5}$ & $.290351 e-1$ & .10 & $.320395 e-1$ & .27 & $.181389 e-1$ & .57 & $.452980 e-2$ & .65 \\
$2^{-6}$ & $.269368 e-1$ & .11 & $.257759 e-1$ & .31 & $.119042 e-1$ & .61 & $.276609 e-2$ & .71 \\
$2^{-7}$ & $.248167 e-1$ & .12 & $.204135 e-1$ & .34 & $.776732 e-2$ & .62 & $.166030 e-2$ & .74 \\
$2^{-8}$ & $.227230 e-1$ & .13 & $.160242 e-1$ & .35 & $.508354 e-2$ & .61 & $.985732 e-3$ & .75 \\
$2^{-9}$ & $.206956 e-1$ & .13 & $.125141 e-1$ & .36 & $.333694 e-2$ & .61 & $.587477 e-3$ & .75 \\
$2^{-10}$ & $.187693 e-1$ & .14 & $.975028 e-2$ & .36 & $.220257 e-2$ & .60 & $.349089 e-3$ & .75 \\
\hline
\end{tabular}

This procedure yields $N$ approximate roots of the polynomial $p_{N}$ in (5.7). Each of these roots leads to a different approximate value of the order $\gamma$ and hence, in the case $N>1$, this introduces an element of choice. Our computational experience suggests that the root corresponding to $\omega^{-1}$ often leads to a substantially more accurate estimate of $\gamma$. The estimates reported in Table 8 were based on that choice. The table indicates that each increment of $N$ improves the rate of convergence by a factor of (roughly) $\gamma$. This seems to be typical of the general case, and we conjecture that this procedure achieves an accuracy of $O\left(R^{(N+1) \gamma}\right)$ for $N<(1-\gamma) / \gamma$.

Assuming that (5.3) holds, we may pursue the numerical analysis of the solution near the singular point still further. Having obtained an estimate for $\gamma$, we can substitute in (5.6) to obtain, after truncation, a linear system for the unknown approximate values of $a_{j}$. The results can then be translated into estimates for the coefficients $c_{j}$ in (5.3) by making use of an estimate for $R$.

\section{Conclusion}

In this paper, we have described a novel approach to numerically deciphering singularities arising from differential equations, which is based on discrete methods employed in the complex domain. We have shown how Lyness's method for the generation of approximate Taylor coefficients may be succesfully combined with standard time-stepping procedures to yield an algorithm for the detection of the nearest singular points. We have also proposed a technique for the estimation of the order of algebraic branch points which uses numerical continuation. 
One of the initial aims of this research project had been to develop numerical techniques for the estimation of the singularity parameters which would perform well without the need to compute excessively close to the singular point. As our numerical experiments show, we have been only partly successful in this respect. Nevertheless, the techniques described have the advantages of flexibility and ease of implementation. They are also amenable to rigorous analysis and should therefore deserve further development. In particular, it would be desirable to generalize those techniques to cater for multidimensional problems.

\section{BIBLIOGRAPHY}

1. G. A. Baker and P. Graves-Morris, Padé approximants. vol. 1, Addison-Wesley, Reading, MA, 1981.

2. M. Berger and R. V. Kohn, A rescaling algorithm for the numerical calculation of blowing-up solutions, Comm. Pure Appl. Math. 41 (1988), 841-863.

3. R. P. Brent and H. T. Kung, Fast algorithms for manipulating formal power series, J. Assoc. Comput. Mach. 25 (1978), 581-595.

4. J. C. Butcher, The numerical analysis of ordinary differential equations, Runge-Kutta methods and general linear methods, Wiley, Chichester, 1987.

5. Y. F. Chang and G. Corliss, Ratio-like and recurrence tests for convergence of series, IMA J. Appl. Math. 25 (1980), 349-359.

6. G. Corliss, On computing Darboux type series analyses, Nonlinear Anal. 7 (1983), 1247 1253.

7. __ Integrating ODE's in the complex plane-pole vaulting, Math. Comp. 35 (1980), 1181-1189.

8. G. Corliss and Y. F. Chang, Solving ordinary differential equations using Taylor series, ACM Trans. Math. Software 8 (1982), 114-144.

9. J. W. Dold, Analysis of the early stage of thermal runaway, Quart. J. Mech. Appl. Math. 38 (1985), 361-387.

10. P. Henrici, Applied and computational complex analysis, vol. 1, Wiley, New York, 1974.

11. F. Hildebrand, Introduction to numerical analysis, McGraw-Hill, New York, 1956.

12. E. Hille, Ordinary differential equations in the complex domain, Wiley, New York, 1976.

13. C. Hunter, personal communication, 1992.

14. C. Hunter and B. Guerrieri, Deducing the properties of singularities of functions from their Taylor series coefficients, SIAM J. Appl. Math. 39 (1980), 248-263.

15. Y. Kimura, Parametric motion of complex-time singularity toward real collapse, Phys. D 46 (1990), 439-448.

16. J. D. Lambert and B. Shaw, A method for the numerical solution of $y^{\prime}=f(x, y)$ based on a selfadjusting non-polynomial interpolant, Math. Comp. 20 (1966), 11-20.

17. H. A. Levine, The role of critical exponents in blowup theorems, SIAM Rev. 32 (1990), 262-288.

18. J. N. Lyness, Differentiation formulas for analytic functions, Math Comp. 21 (1967), 352362.

19. D. I. Meiron, G. R. Baker, and S. Orszag, Analytic structure of vortex sheet dynamics. Part 1. Kelvin-Helmholtz instability, J. Fluid Mech. 114 (1982), 283-298.

20. T. Nakagawa, Blowing up of a finite difference solution to $u_{t}=u_{x x}+u^{2}$, Appl. Math. Optim. 2 (1976), 337-350.

21. L. E. Payne, Improperly posed problems in partial differential equations, SIAM, Philadelphia, PA, 1975.

22. B. Shaw, Modified multistep methods based on a nonpolynomial interpolant, J. Assoc. Comput. Mach. 14 (1967), 143-154. 
23. C. Sulem, P. L. Sulem, and H. Frisch, Tracing complex singularities with spectral methods, J. Comput. Phys. 50 (1983), 138-161.

24. Y. Tourigny and J. M. Sanz-Serna, The numerical study of blowup with application to a nonlinear Schrödinger equation, J. Comput. Phys. 102 (1992).

School of Mathematics, University of Bristol, Bristol, England

E-mail address: Y.Tourigny@uk.ac.bristol

Department of Engineering Mathematics, University of Bristol, Bristol, England E-mail address: M.GrinfeldQuk.ac.bristol 